\title{
Ein Job wie jeder andere?
}

\section{B. Gurtner}

1 Schneider S, Winistörfer M, Glauser R. Viel Arbeit für weniger Arbeit. Schweiz Ärztezeitung 2003; 84(42):2189-90.

Korrespondenz:

Dr. med. Bernhard Gurtner

Eggstrasse 76

CH-8620 Wetzikon
Wissen Sie noch, weshalb Sie den Arztberuf gewählt haben? Je länger diese Entscheidung zurückliegt, um so mehr dürften wir uns über die wahren Beweggründe täuschen. Wurden wir getrieben durch naturwissenschaftliche Neugier, manuelle Begabung, jugendlichen Helferdrang, Familientradition oder durch die uneingestandene Lust nach Geld und Geltung? Waren wir mit 20 Jahren Idealisten, die Mitmenschlichkeit zum eigenen Beruf machen wollten, und mit 30 bereits illusionsfreie Pragmatiker, abgebrüht durch manche Erfahrungen an unseren Aus- und Weiterbildungsstätten? Welche beruflichen Vorstellungen und Wünsche haben sich erfüllt, und was ist infolge der stürmischen sozialen Veränderungen rettungslos über Bord gespült worden? Befriedigt Sie das Arztsein noch - intellektuell, emotionell, finanziell - oder schon lange in keiner Weise mehr?

Die Pflegeberufe haben sich schneller als das ärztliche Patriarchat den neuen Gegebenheiten angepasst. Die alten Ordensschwestern, die sich im Jenseits und für alle Ewigkeit den Gotteslohn für ihren selbstlosen Einsatz erhofften, wurden durch junge Frauen abgelöst, die hier und jetzt bessere Arbeitsbedingungen verlangten. Berufung wurde durch Beruf ersetzt, liebevolle $\mathrm{Zu}$ wendung als angelerntes Verhalten in Frage gestellt. «Pflege ist ein Job wie jeder andere» diente als Hauptargument im Kampf für mehr professionelle Autonomie und Anerkennung, gerechte Löhne und für die 42-Stunden-Woche. Von der verfügbaren Zeit wird höchstens noch ein Viertel für die Berufstätigkeit eingeplant, man (frau) will nicht mehr rund um die Uhr mit dem Spital und seinen Problemen verheiratet sein.

Die Assistenzärztinnen und Assistenzärzte sind noch nicht ganz soweit, doch wurde ihnen nach Beschluss der eidgenössischen Räte versprochen, die wöchentliche Höchstarbeitszeit ab 1. Januar 2005 auf 50 Stunden zu beschränken. Das neue Gesetz enthält auch grosszügige Ruhezeit- und Kompensationsregelungen; Pausen und nächtlicher Pikettschlummer gelten voll als Arbeitszeit. Die nun sehr knapp gewordenen Weiterbildungsstunden liessen sich vielleicht wiedergewinnen, wenn man den Schlafenden aktuelles Fachwissen über Kopfhörer hypnopädeutisch einflüstern könnte. Behandlungsfehler wegen Übermüdung sollte es keine mehr geben, dafür werden sich ebenso folgenschwere Informationspannen häufen, weil mehrmals täglich übergeben und rapportiert werden muss. Wie der
Verband Schweizerischer Assistenz- und Oberärztinnen und -ärzte VSAO prophylaktisch gewarnt hat [1], kommt da viel Arbeit für weniger Arbeit auf die Spitalleitungen zu.

Es müssen aber nicht nur Stellen- und Dienstpläne angepasst werden. Auch das ärztliche Selbstverständnis und dessen Wahrnehmung in der Öffentlichkeit werden sich wandeln. Der Eindruck könnte sich verstärken, dass die immer zahlreicheren Ärztinnen und Ärzte nur einen Job wie jeden anderen ausüben. Das brächte keinen Schaden für ihr Verhältnis zu mündig gewordenen Kundinnen und Kunden, die nur für Gelenkreparaturen oder zwecks Behebung harmloser Gesundheitsstörungen vorsprechen. Sobald aber ein Mensch in einer lebensbedrohenden Situation nach Hilfe verlangt, sucht er eine verlässliche Bezugsperson, die ihm ein durchrationalisierter «Job wie jeder andere» nur in einem rasch wechselnden Team und in vielfach fragmentierter Teilzeit anbieten kann.

Die Angst vor Leiden und Tod und die Hoffnung auf Rettung stellen die Heilkundigen in den Augen der Kranken auch heute noch auf ein von mystischem Weihrauch umwehtes Podest, von dem viele moderne Mediziner gerne heruntersteigen möchten. Wie hoch der Arztberuf trotz fortschreitender Selbstdemontage immer noch eingeschätzt wird, lässt sich aber daran erkennen, wie beliebt Arztserien im Fernsehen und Arztromane am Kiosk sind. Wer diesen Kitsch nicht in $\mathrm{Zu}$ sammenhang mit seinem Berufsbild bringen möchte, verkennt die verbreitete und tiefliegende Sehnsucht nach charismatischen Heilern. Wenn dieses Anliegen von den arbeitsteilig jobbenden Teams nicht wahrgenommen wird, kann sich keine vertrauensvolle therapeutische Beziehung entwickeln. Der frustrierte Patient sucht sich einen geduldigen und verständnisvollen Naturarzt oder eine fernöstlich inspirierte Therapeutin.

Sicher wird es auch im rechtlich reglementierten «Job wie jeder andere» immer wieder einige «Handholder» geben, die sich den Kranken über den vertraglichen Dienstschluss hinaus ganz persönlich zuwenden und verantwortlich fühlen. Das bringt den medizinischen Spitalangestellten einen beglückenden Teil von dem, was sie sich bei der Berufswahl einmal gewünscht hatten. Dieses oft etwas belächelte Helfersyndrom muss ja nicht gleich wieder zu einer 70-Stunden-Woche ausarten, es könnte aber jener Sinnentleerung vorbeugen, von der alle extrem spezialisierten Jobs bedroht sind. 